\title{
Myelopathy: chameleons and mimics
}

\author{
Lionel Ginsberg
}

Department of Clinical

Neurosciences, UCL Institute of

Neurology, Royal Free Campus,

University College London,

London, UK

\section{Correspondence to}

Professor Lionel Ginsberg, Department of Neurology, Royal

Free Hospital, Pond Street, London NW3 2QG, UK;

Lionel.Ginsberg@nhs.net

Accepted 2 September 2016

Published Online First

21 November 2016

\begin{abstract}
The diagnosis of spinal cord disease may be delayed or missed if the presentation does not conform to the expected pattern of a

symmetrical spastic paraparesis with sphincter dysfunction and a sensory level. This may occur when a myelopathy has yet to evolve fully, or is highly asymmetrical, as in Brown-Séquard syndrome. Other potential distractions include fluctuating symptoms, as may accompany spinal cord demyelination, and pseudoneuropathic features, as seen acutely in spinal shock and in the chronic setting with some high cervical cord lesions. A second pathology, such as a polyneuropathy or polyradiculopathy, can mask the presence of a myelopathy. The converse situation, of non-myelopathic disease mimicking a cord lesion, arises typically when symptoms and/or signs approximate bilateral symmetry. This may happen with certain diseases of the brain, or of the peripheral nerves, with functional disorders and even occasionally with nonneurological disease. These sources of diagnostic difficulty assume clinical importance when they delay the recognition of conditions that require urgent treatment.
\end{abstract}

\section{INTRODUCTION}

The key anatomical property of the spinal cord is its small cross-sectional area, within which are located, in close proximity, ascending and descending tracts relating to both sides of the body, the whole being housed within the narrow confines of the bony spinal column (figure 1). This structure usually dictates a 'default' clinical presentation of myelopathy, with little room for diagnostic confusion when fully evolved-upper motor neurone (UMN) features in both lower limbs, variable involvement of the upper limbs depending on the site of the lesion (rostral to caudal, and intrinsic vs extrinsic), a sensory level and sphincter disturbance. $^{1}$

Difficulty recognising that a patient has a myelopathy (a myelopathy chameleon) arises with incomplete cord syndromes, particularly if there are unilateral or highly asymmetrical clinical features. Other potential distractions include the presence of pseudo lower motor neurone (LMN) signs or pseudoneuropathic symptoms. Myelopathies resulting from lesions of the most rostral ${ }^{2}$ or most caudal ${ }^{3}$ portions of the cord can produce atypical and misleading clinical findings because the arrangement of the tracts becomes irregular in these regions. Sometimes, a myelopathy may be masked by signs arising from a second neuropathological process. Finally, diagnostic insecurity may occur when initial spinal imaging is normal. ${ }^{4}$

The converse state of affairs, of a patient mistakenly being labelled as having a myelopathy (a myelopathy mimic), generally happens when a nonmyelopathic neurological disease, or even non-neurological condition, presents with real or apparent bilateral symptoms and signs, particularly UMN signs. In the acute setting, the imperative of rapidly excluding cord compression may lead to neurosurgical referral and urgent spinal imaging, even for patients without hard UMN signs, and hence with a relatively low level of suspicion of myelopathy. This is somewhat analogous to the management of stroke mimics, ${ }^{5}$ where time constraints can mean a patient at low actual stroke risk is hurried down a pathway that includes urgent brain imaging and perhaps even consideration of thrombolysis.

\section{MYELOPATHY CHAMELEONS}

Because standard spinal imaging usually (though not invariably) detects a structural cord lesion when one is present, it is logical first to consider scenarios where requesting this investigation might be delayed (myelopathy chameleons-table 1).

\section{The history}

It is a truism that the history depends upon who takes it. If a patient with myelopathy presents to the wrong hospital 


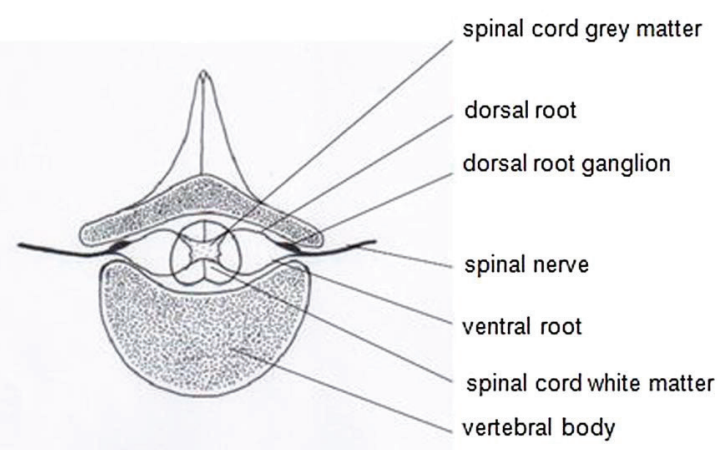

Figure 1 Diagram of a transverse section of the spinal cord within the spinal canal at mid-thoracic level.

Table 1 Myelopathy chameleons

\begin{tabular}{|c|c|}
\hline $\begin{array}{l}\text { Myelopathy } \\
\text { masquerading as.... }\end{array}$ & Mechanism.... \\
\hline Stroke & $\begin{array}{l}\text { Brown-Séquard syndrome with predominant } \\
\text { unilateral weakness }\end{array}$ \\
\hline Motor neurone disease & Infiltrative myeloradiculopathy \\
\hline Lumbar polyradiculopathy & $\begin{array}{l}\text { Exercise-dependent symptoms resembling } \\
\text { lumbar canal stenosis or conus lesion with } \\
\text { predominant LMN signs }\end{array}$ \\
\hline Brachial neuritis & $\begin{array}{l}\text { Monomelic presentation of an intrinsic or } \\
\text { very high cervical cord lesion }\end{array}$ \\
\hline Acute polyneuropathy & Spinal shock \\
\hline Chronic polyneuropathy & $\begin{array}{l}\text { Incomplete evolution of a sensory level or } \\
\text { masking of myelopathy by a neuropathy } \\
\text { (dual pathology) }\end{array}$ \\
\hline Restless legs syndrome & Nocturnal leg spasms \\
\hline Metabolic myopathy & Exercise-dependent symptoms \\
\hline Peripheral vascular disease & Exercise-dependent symptoms \\
\hline $\begin{array}{l}\text { Osteoarthritis (hip, knee, } \\
\text { lumbar) }\end{array}$ & Misinterpretation of history \\
\hline
\end{tabular}

LMN, lower motor neurone.

department, the working diagnosis may simply reflect the prejudices of the attending doctor. For example, an orthopaedic surgeon may focus on an osteoarthritic hip or knee joint, or perhaps lumbosacral spondylosis, as the explanation of a patient's difficulty walking, without recognising that similar degenerative processes are affecting the patient's cervicothoracic spine. While orthopaedic surgeons are often wise to this differential diagnosis nowadays, many neurologists will have seen patients whose gait disorder was unrelieved by hip or knee replacement, because the dominant cause was cervical spondylotic myelopathy all along.

Other sources of confusion in the history include the tendency of some cord lesions to produce fluctuating symptoms. This is particularly marked with spinal cord demyelination, where exercise-induced symptom exacerbation, a manifestation of Uhthoff's phenomenon, ${ }^{6}$ can be misinterpreted as arising from peripheral vascular disease, lumbar canal stenosis or even a

\section{Box 1 Exercise-induced symptoms}

A 56-year-old man presented with a 6-month history of exercise-induced leg weakness. After walking for $10 \mathrm{~min}$, his legs would become weak, worse on the left, and he would have to stop. There was no pain or sphincter disturbance. He had a history of hypertension and was a smoker. On examination, pedal pulses were present. He had a pale left optic disc and a left relative afferent pupillary defect. After walking for $10 \mathrm{~min}$, his left leg became weak and he had unsustained clonus at the left ankle, with an upgoing left plantar response.

Comment: This patient was originally thought to have peripheral vascular disease, because of the relationship of the symptoms to exercise and the vascular risk factors, despite the absence of pain. He had been referred via the vascular department but the clinical findings were ultimately correctly ascribed to spinal cord demyelination, the exercise-induced exacerbation being a manifestation of Uhthoff's phenomenon. There were also signs of previous left optic neuritis and the patient subsequently recalled an episode of left-sided blurred vision lasting 1 week, 20 years earlier.

metabolic myopathy (box 1). Fluctuating symptoms, again with a relationship to exercise, also occur with some spinal vascular malformations, such as a dural arteriovenous fistula, typically encountered in middle-aged and elderly men. Reaching the correct diagnosis in these patients may be further confounded by failure to detect an abnormality on standard spinal MRI sequences. ${ }^{4} 7$ Nocturnal symptom fluctuation may also be misleading, leg spasms arising from UMN involvement in myelopathy occasionally being mistaken for 'restless legs'.

Recognising that a patient has a myelopathy from the history can be especially difficult when a cord syndrome has only partially evolved. The spinothalamic tracts are somatotopically organised in such a way that fibres relating to the sacral and lumbar dermatomes are located close to the surface of the cord whereas those relating to thoracic and cervical dermatomes are found more centrally (figure 2). Thus, an extrinsic compressive lesion, say of the thoracic cord, may initially preferentially affect the lumbosacral dermatomes and only give rise to a sensory level on the trunk as the condition progresses. ${ }^{9} 10$ Patients may actually describe sensory loss ascending with time from the feet to the legs and then to the abdomen. The diagnostic problem arises when a patient presents before a truncal sensory level has fully evolved, in which case the sensory symptoms may approximate a stocking distribution, mimicking a sensory neuropathy. With some high cervical cord lesions, there can be a more confusing clinical picture still, with glove-and-stocking numbness, an even closer neuropathy mimic. 


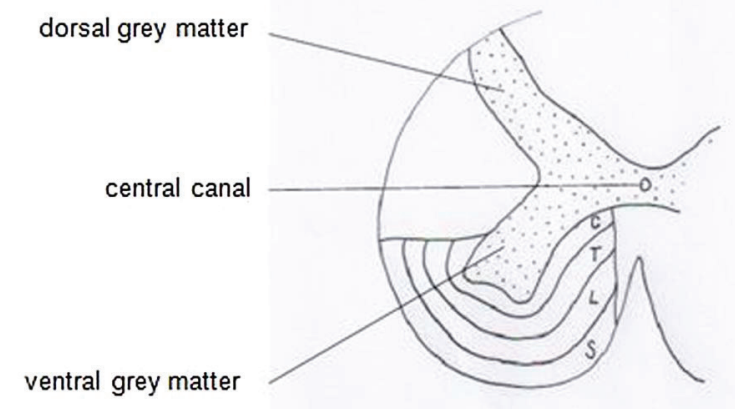

Figure 2 Diagram of a transverse section of the cervical spinal cord, showing the somatotopic organisation of the spinothalamic tracts (schematically enlarged), with fibres relating to the sacral dermatomes $(\mathrm{S})$ located most superficially and the lumbar $(\mathrm{L})$, thoracic $(\mathrm{T})$ and cervical $(\mathrm{C})$ dermatomes being represented at successively deeper levels within the cord white matter (adapted from Standring et $a l^{8}$ ).

\section{Box 2 Brown-Séquard syndrome}

A 67-year-old woman presented to a hyperacute stroke unit, with an abrupt onset of left arm and leg weakness. She had a past history of type 2 diabetes mellitus and hypertension, and had a permanent pacemaker. On examination, there was grade 3 weakness of the left arm and leg. The supinator reflexes were inverted. Pinprick sensation was reduced in the right leg and there was impairment of joint position sensation at the toes on the left.

Comment: This patient's sensory features only became apparent on examination. With the abrupt onset of unilateral weakness and the presence of vascular risk factors, activation of the hyperacute stroke pathway was understandable. Matters were further complicated by the presence of a pacemaker, which was MRI-incompatible. CT cranial scan was unremarkable. CT of cervical spine showed an extruded calcified disc fragment occupying the left side of the spinal canal at C5/6, consistent with the abrupt onset, Brown-Séquard syndrome and inverted supinator reflexes. The patient gradually improved following urgent decompressive surgery.

\section{Incomplete cord syndromes}

Most myelopathies are incomplete, even when the history has fully evolved, and can therefore be mistaken for alternative pathologies.

Hemicord lesions

A fully fledged Brown-Séquard syndrome arising from cord hemisection is a rarity nowadays. Far likelier is a partial lesion, with symptoms and signs on one side of the body dominating the clinical picture. If a patient

\section{Box 3 Early syringomyelia}

A 19-year-old woman presented with a 3-month history of pain and weakness in the right arm. There were no sensory symptoms. On examination, there was mild, predominantly distal, patchy wasting and weakness of the right arm. Tendon reflexes were absent in that limb and the superficial abdominal responses were also absent on the right. The remaining tendon reflexes were normal and the plantar responses were downgoing. Pinprick sensation was impaired on the right from C4 to $\mathrm{T} 5$, with preservation of joint position sensation in the right arm.

Comment: This patient was initially thought to have brachial neuritis. However, the relatively subtle sensory signs, of a suspended nociceptive sensory level with preserved posterior column function, and the unilateral absent abdominal responses, indicated a more extensive process, most likely an intrinsic cord lesion. This was confirmed by spinal MRI which showed syringomyelia with an associated Chiari I malformation. As in case 2, the lack of sensory symptoms was potentially misleading.

develops unilateral weakness of abrupt onset and contralateral sensory features are underplayed, there is a risk that the presentation may mimic a stroke (box 2).

Intrinsic cord lesions

The clinical features of advanced syringomyelia do not usually cause diagnostic dilemmas but milder and earlier disease can be misleading, for example, with monomelic presentations and a predominance of LMN signs (box 3).

Rostral and caudal syndromes

In addition to pseudoneuropathic sensory symptoms, a high cervical cord lesion can give rise to apparent LMN signs, with distal wasting and weakness in the upper limbs, for reasons that are ill-understood, but possibly vascular in basis. Lesions at the uppermost limit of the cervical cord, at foramen magnum level, can be misleading, with initial monomelic clinical signs, only later progressing to involvement of the other limbs, in a rotating sequence (eg, right arm then right leg, then left leg, then left arm). At the lower end of the spinal cord, 'conus' lesions typically produce a mixture of $\mathrm{LMN}$ and $\mathrm{UMN}$ signs. The LMN signs may predominate, however, leading to a misdiagnosis of cauda equina syndrome, ${ }^{3}$ with the risk that a structural lesion a little further up the neuraxis is missed.

\section{Spinal shock}

The term 'spinal shock' refers to the hyperacute phase of a massive spinal cord injury, when spasticity has not yet developed and tendon reflexes are lost, paradoxically. ${ }^{11}$ This pattern of signs can persist for days or even weeks, before tone increases and hyper- 
reflexia evolves, and may be mistaken for an acute neuropathy. Because an acute cord lesion can present in this way, there is no chagrin in requesting an urgent spinal MRI in a patient with presumed Guillain-Barré syndrome. The two conditions can mimic each other quite closely clinically, provided the neuropathy has not (yet) involved the cranial nerves, and every effort must be made to avoid missing an acute treatable cord lesion. That said, most pathological processes that give rise to spinal shock, for example, irreversible trauma, extensive infarction, acute necrotising myelopathy, do not require urgent neurosurgical intervention.

\section{Dual pathology}

A severe peripheral neuropathy may mask signs of a coexistent myelopathy. The two processes may be unrelated, as with a patient with a diabetic neuropathy who develops myelopathy secondary to cervical spondylosis - both relatively common conditions-the presence of the diabetic neuropathy potentially delaying the diagnosis of the cervical myelopathy. Sometimes, however, the same pathological process can injure both the spinal cord and the peripheral nerves. Vitamin $\mathrm{B}_{12}$ deficiency is perhaps the best known example, with part of the myelopathy and part of the neuropathy resulting from, at a cellular level, a distal axonopathy of the central and peripheral projections, respectively, of the same sensory neurone(s). This combination of neuropathy and myelopathy is one of the causes of the time-honoured neurological 'paradox' of absent ankle reflexes with upgoing plantar responses. Vitamin $\mathrm{B}_{12}$ deficiency does not usually cause diagnostic confusion in this context, since serum $\mathrm{B}_{12}$ estimation is high on the list of 'screening' investigations for causes of both neuropathy and myelopathy. But recognition of a myelopathic contribution in a patient with a peripheral neuropathy can be more important in raising the level of suspicion of rarer metabolic disorders, which might not otherwise be investigated, such as functional vitamin $B_{12}$ deficiency ${ }^{12}$ or hypocupraemic myeloneuropathy. ${ }^{13}$

A particularly misleading situation can arise when a neoplastic process simultaneously affects the spinal cord and the motor roots, causing a mixture of LMN and UMN signs in all four limbs. This pattern typically occurs when epidural metastases from prostate cancer compress the cord at multiple levels and infiltrate the roots. Lymphoma may have similar effects, but is usually more painful and less insidious. If sensory signs are minimal or non-existent, the patient may be assumed to have motor neurone disease. The possibility of a structural lesion of this kind is one of the reasons spinal imaging is mandatory in patients with suspected motor neurone disease with clinical features confined to the limbs, even when there is a combination of LMN and UMN signs in the lower limbs.
Table 2 Myelopathy mimics

Actual site of

\begin{tabular}{ll} 
lesion... & Pathological mechanism... \\
\hline $\begin{array}{l}\text { Central nervous } \\
\text { system }\end{array}$ & $\begin{array}{l}\text { Diffuse UMN disorder (genetic, } \\
\text { developmental, degenerative, } \\
\text { immunological) }\end{array}$ \\
& Multifocal brain disease (vascular, \\
& neoplastic) \\
Peripheral nervous & Unifocal brain lesion (vascular, neoplastic) \\
system & Lumbar polyradiculopathy \\
Non-neurological & Neuropathy, eg, Guillain-Barré syndrome \\
Functional & Metabolic myopathy \\
& $\longrightarrow$ Endocrine
\end{tabular}

UMN, upper motor neurone.

\section{MYELOPATHY MIMICS}

Myelopathy mimics are best classified according to the true site of the lesion that is being misinterpreted as myelopathic, which may be neurological or nonneurological (table 2).

\section{Central nervous system}

Disorders of the central nervous system that mimic myelopathies may exert a diffuse effect on UMNs, or may be multifocal, or rarely unifocal.

Several neurogenetic, neurodevelopmental and neurodegenerative conditions diffusely target UMNs, or appear to do so, selectively or in association with other neurological deficits, thereby potentially mimicking a structural cord lesion. Among genetic disorders, hereditary spastic paraplegias (HSP) and adrenomyeloneuropathy are perhaps the most obvious mimics. But patients with Friedreich's ataxia can present with a predominant spastic paraparesis, resembling a myelopathy. This is especially likely to occur in people with at least one relatively small GAA expansion, ${ }^{14}$ or in compound heterozygotes with particular missense point mutations in one allele. ${ }^{15}$ Spinocerebellar ataxias can also occasionally present with prominent UMN signs, ${ }^{16}$ but are more likely to be misdiagnosed as HSP than a structural myelopathy, given the increased likelihood of a family history. From a pragmatic standpoint, the most important inherited disorder to consider in younger patients with an apparent myelopathy and negative spinal imaging, particularly when there is diurnal fluctuation, is dopa-responsive dystonia, because of the therapeutic implications. ${ }^{17}$ Neurodevelopmental disorders which can cause diagnostic confusion include diplegic cerebral palsy, particularly if mild, with UMN features only coming to clinical attention in adolescence or early adult life.

Diffuse neurodegenerative disorders targeting UMNs, or seeming to do so, include primary lateral 
sclerosis (and indeed motor neurone disease), multiple system atrophy with mainly pyramidal features, and even progressive supranuclear palsy, if a significant eye movement disorder has not yet evolved. Because these conditions are all bilateral, albeit sometimes asymmetrical, they can mimic a myelopathy. In practice, a request for spinal imaging contributes little to diagnostic delay in these patients, and will often have been included in the workup anyway, to ensure that treatable conditions have not been missed.

Similarly, identification of the stiff person syndrome, an immunological rather than degenerative diffuse disorder, will not be unduly delayed by negative spinal imaging, but depends rather on the recognition that the patient's stiffness and spasms do not represent the spasticity of a pyramidal disorder, and that there are unusual signs for myelopathy, such as an exaggerated lumbar lordosis. The characteristic electrodiagnostic finding of continuous motor unit activity and serological finding of antiglutamic acid decarboxylase antibodies will then help clinch the diagnosis. ${ }^{18}$

Multifocal structural and vascular brain lesions can be more challenging. Bihemispheric small vessel disease is often initially mistaken for a myelopathy, until realisation dawns that the patient has a 'gait apraxia' rather than a true spastic paraparetic gait, and that there are features beyond what would be expected for a UMN lesion-cognitive impairment and signs of frontal lobe release. Likewise, but with a more rapidly progressive course, multiple cerebral metastases can present with predominant bilateral UMN signs, but other features are usually found on careful examination.

Unifocal structural brain lesions masquerading as a myelopathy are unusual. They can occasionally occur in the brainstem, where, for example, a vascular malformation can exert effects on the long tracts that overshadow any cranial nerve involvement. Perhaps the best known solitary brain lesion to mimic a myelopathy is a parasagittal or parafalcine meningioma.

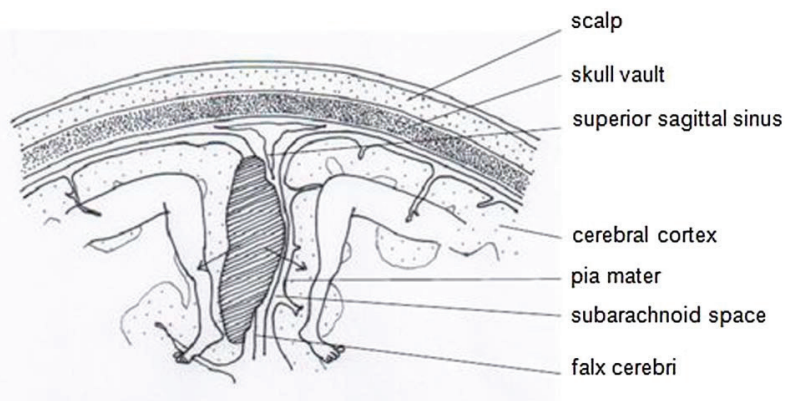

Figure 3 Diagram of a coronal section across the midline of the skull vault, showing a parasagittal mass (hatched) compressing (arrows) the representation of the leg in the motor cortex bilaterally (motor homunculus adapted from Penfield and Rasmussen ${ }^{19}$ ).
This mass adjacent to or on the falx can compress the representation of the leg in the motor cortex bilaterally, resulting in a spastic paraparesis, albeit often asymmetrical (figures 3 and 4). In the pre-MRI era, this was a potentially dangerous situation, the patient undergoing myelography for a presumed cord lesion, with a risk of coning. There can be a clinical clue that a patient's spastic paraparesis is arising from a parasagittal meningioma rather than a myelopathy, namely the detection of an associated bony exostosis on inspection and palpation of the skull. Superior sagittal sinus thrombosis is another midline intracranial disorder that can mimic a myelopathy. Here, the parafalcine damage, to the 'leg' of the motor homunculus in the anterior paracentral lobule bilaterally, results from venous congestion and oedema, sometimes with frank infarction and haemorrhage, rather than compression.

\section{Peripheral nervous system}

While it is possible to envisage an acute metabolic myopathy, such as a periodic paralysis, mimicking an acute myelopathy, the main peripheral disorder where an acute cord lesion enters the differential diagnosis is Guillain-Barré syndrome (see above-Spinal shock). This will only be the case when the neuropathy has not (yet) involved cranial nerve territory, and is likelier with paraparetic presentations, ${ }^{20}$ particularly with retained reflexes. ${ }^{21}$

Moving more centrally, but still technically in the peripheral nervous system, lumbosacral spondylotic polyradiculopathy can resemble a myelopathy, given the bilateral clinical features, albeit exclusively LMN. A more common clinical dilemma arises with neural compromise at more than one site, that is, with coexistent lumbar and cervical spondylosis, making it difficult to determine the major contributor to a patient's disability.

\section{Non-neurological conditions}

The hyper-reflexia of thyrotoxicosis and the pseudomyelopathic features that can occasionally accompany metabolic disorders such as hypocalcaemia are sometimes prominent enough to trigger a request for spinal imaging. In reality, this is not generally a source of significant diagnostic delay, as the salient blood tests will probably have been requested at the same time.

\section{Functional neurological symptoms}

Though nowadays relatively rare, functional weakness of both legs, mimicking a myelopathy, can be a manifestation of a conversion disorder, ${ }^{22}$ previously termed hysterical paraplegia ${ }^{23}$ (box 4). The clinical context, along with the presence of positive features of functional disease (and absence of 'hard' signs), should raise the suspicion of a non-organic problem. Such positive features could include disparities in muscle strength when tested in different ways (eg, on 


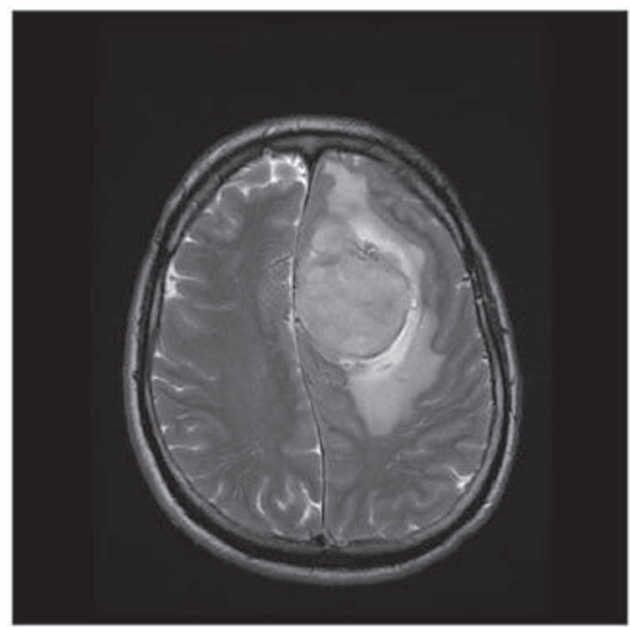

Figure 4 Axial T2-weighted MRI scan of brain showing a parafalcine meningioma.

Box 4 Conversion disorder (adapted from Ginsberg) $^{24}$

A 22-year-old woman was unable to move her legs on recovering from a minor gynaecological operation. Continence was preserved. Her legs felt numb. On examination, both legs were apparently completely paralysed but tone, tendon reflexes and plantar responses were all normal. There was a non-anatomical distribution of cutaneous sensory loss and errors occurred on testing proprioception at a rate greater than would be expected by chance.

Comment: The context of this patient's presentation, along with the lack of objective neurological signs and some positive non-organic signs, suggested a functional basis. Immediate MRI of the neuraxis was normal, as were electromyography and nerve conduction, and the patient responded to combined psychological and physiotherapeutic intervention, during the course of which a complex psychosocial and psychosexual background emerged.

the couch vs attempted standing and walking), and a non-anatomical distribution of sensory impairment. If paralysis is incomplete, Hoover's sign can be helpful. ${ }^{25}$ Spinal imaging is appropriate at the outset, partly to allay patient (and doctor) anxieties, and partly to exclude the remote possibility of organic 'underlay'. Once a structural myelopathy has been ruled out and a positive diagnosis of functional disorder made, however, repeated investigation should be avoided as it sends the patient mixed messages, and there should be an early shift in focus to management. ${ }^{26}$

\section{CONCLUSION}

When a patient's history or examination deviates from the default or standard pattern expected from a cord lesion-spastic paraparesis, sensory level, sphincter disturbance-there is the possibility of diagnostic delay. Conversely, in situations where there is heightened anxiety about cord compression, patients with clinical features only superficially resembling those of a myelopathy may be hurried down an inappropriate pathway. Many of the difficulties of underdiagnosis and overdiagnosis of myelopathy have been described in this article. The most important are those where there is a delay in recognising conditions that require urgent treatment.

\section{Key points}

- Cord lesions which have not completely evolved, or which are highly asymmetrical, or which produce fluctuating symptoms, may be subject to diagnostic delay.

- A myelopathy may also be masked by a second process, such as a polyneuropathy or polyradiculopathy.

- Lesions outside the cord that give rise to bilaterally symmetrical symptoms and/or signs may be mistaken for a myelopathy.

- These misdiagnoses are important if they delay treatment, for example, treatment of surgically amenable cord lesions in myelopathy chameleons, and of lesions that can be resected from other sites in myelopathy mimics. In some cases, urgent medical management may also be delayed.

Acknowledgements The author thanks his wife Sue Ginsberg, for preparing the diagrams.

Disclaimer The case histories in this article are each amalgams of several patients and also include fictional elements, rather than being based on individuals, to preserve anonymity and to aid emphasis of specific points.

Competing interests None declared.

Provenance and peer review Commissioned; externally peer reviewed. This paper was reviewed by Ashwin Pinto, Southampton, UK.

\section{REFERENCES}

1 Ginsberg L. Disorders of the spinal cord and roots. Pract Neurol 2011;11:259-67.

2 Carpenter MB. Core text of neuroanatomy. 4th edn. Baltimore, MD: Williams \& Wilkins, 1991:118.

3 Brodal A. Neurological anatomy in relation to clinical medicine. 3rd edn. New York, NY: Oxford University Press, 1981:778.

4 Wong SH, Boggild M, Enevoldson TP, et al. Myelopathy but normal MRI: where next? Pract Neurol 2008;8:90-102.

5 Fernandes PM, Whiteley WN, Hart SR, et al. Strokes: mimics and chameleons. Pract Neurol 2013;13:21-8. 
6 Ferrari MD, Hilkens PH, Kremer B, et al. Intermittent pyramidal claudication as presenting and sole symptom in multiple sclerosis. J Neurol Neurosurg Psychiatr 1988;51:147-8.

7 Saraf-Lavi E, Bowen BC, Quencer RM, et al. Detection of spinal dural arteriovenous fistulae with MR imaging and contrast-enhanced MR angiography: sensitivity, specificity and prediction of vertebral level. AJNR Am J Neuroradiol 2002;23:858-67.

8 Standring S, Ellis H, Healy JC, et al, eds. Gray's anatomy. 39th edn. Edinburgh, UK: Elsevier, 2005:318.

9 Brodal A. Neurological Anatomy in relation to Clinical Medicine. $3^{\text {rd }}$ edn. New York, NY: Oxford University Press, 1981;75:135.

10 Jamieson DR, Teasdale E, Willison HJ. False localising signs in the spinal cord. BMJ 1996;312:243-4.

11 Brodal A. Neurological Anatomy in relation to Clinical Medicine. $3^{\text {rd }}$ edn. New York, NY: Oxford University Press, 1981:250-1.

12 Turner MR, Talbot K. Functional vitamin B12 deficiency. Pract Neurol 2009;9:37-41.

13 Kumar N, Gross JB Jr, Ahlskog JE. Copper deficiency myelopathy produces a clinical picture like subacute combined degeneration. Neurology 2004;63:33-9.

14 Coppola G, De Michele G, Cavalcanti F, et al. Why do some Friedreich's ataxia patients retain tendon reflexes? A clinical, neurophysiological and molecular study. J Neurol 1999;246:353-7.

15 Cossée M, Dürr A, Schmitt M, et al. Friedreich's ataxia: point mutations and clinical presentation of compound heterozygotes. Ann Neurol 1999;45:200-6.
16 Kaneko A, Narabayashi Y, Itokawa K, et al. A case of Machado-Joseph disease presenting with spastic paraparesis. J Neurol Neurosurg Psychiatr 1997;62:542-3.

17 Németh AH. The genetics of primary dystonias and related disorders. Brain 2002;125:695-721.

18 Barker RA, Revesz T, Thom M, et al. Review of 23 patients affected by the stiff man syndrome: clinical subdivision into stiff trunk (man) syndrome, stiff limb syndrome, and progressive encephalomyelitis with rigidity. J Neurol Neurosurg Psychiatr 1998;65:633-40.

19 Penfield W, Rasmussen T. The cerebral cortex of man. New York, NY: Macmillan, 1950:57.

20 van den Berg B, Fokke C, Drenthen J, et al. Paraparetic Guillain-Barré syndrome. Neurology 2014;82:1984-9.

21 Yuki N, Kokubun N, Kuwabara S, et al. Guillain-Barré syndrome associated with normal or exaggerated tendon reflexes. J Neurol 2012;259:1181-90.

22 American Psychiatric Association. Diagnostic and Statistical Manual of Mental Disorders. 5th edn. (DSM- $5^{\mathrm{TM}}$ ). Arlington, VA: American Psychiatric Association, 2013:318-21.

23 Baker JH, Silver JR. Hysterical paraplegia. J Neurol Neurosurg Psychiatr 1987;50:375-82.

24 Ginsberg L. Lecture notes on neurology. 9th edn. Chichester, UK: Wiley-Blackwell, 2010:172.

25 Stone J. Functional symptoms in neurology. Pract Neurol 2009;9:179-89.

26 Stone J. Functional neurological disorders: the neurological assessment as treatment. Pract Neurol 2016;16:7-17. 